
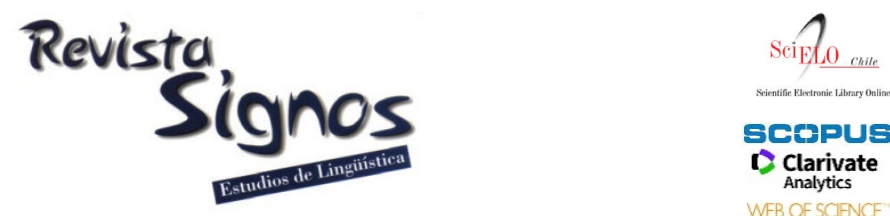

\title{
Aplicación de la Lingüística Sistémico- Funcional al análisis de conferencias académicas*
}

\section{Systemic Functional Linguistics and its Application to the Study of Academic Conference Presentations}

\section{Carolina Viera}

BOISE STATE UNIVERSITY

ESTADOS UNIDOS

carolinaviera@boisestate.edu
Maite Taboada

SIMON FRASER UNIVERSITY

CANADÁ

mtaboada@sfu.ca

Recibido: 08-X-2019 / Aceptado: 09-X-2020

DOI: $10.4067 /$ S0718-09342021000100277

\section{Resumen}

Las comunicaciones en conferencias académicas (CAs) permiten estudiar tanto los rasgos del lenguaje académico oral como las convenciones sociales que se establecen durante estos eventos (Ventola, Shalom \& Thompson, 2002). Los estudios empíricos sobre conferencias son escasos (Robles Garrote, 2016), especialmente con el marco teórico de la Lingüística Sistémico-Funcional (LSF). Este hecho genera que los investigadores que analizan estos textos orales cuenten con escasa bibliografía de referencia. Este trabajo aporta al conocimiento sobre las CAs al discutir aspectos teóricos y metodológicos pertinentes al análisis de un corpus de 32 presentaciones académicas en español realizadas en los Estados Unidos. El marco teórico de la LSF se usa para la determinación de la estructura genérica o estructura prototípica de los textos del corpus, proponiéndose la idoneidad del modelo de macro-género y de estructura orbital (Martin \& Rose, 2008) para su estudio. El análisis evidenció una estructura distintiva de las CAs, diferencias de acuerdo a la disciplina y membresía en la comunidad académica. En este sentido, este estudio contribuye a reducir la brecha existente en esta área del conocimiento y ofrece propuestas de aplicación de la LSF a futuros investigadores.

Palabras Clave: Lingüística Sistémico-Funcional, estructura genérica, macro-género, español académico oral, español en los EE.UU. 


\begin{abstract}
Academic conference presentations (CPs) offer the possibility to study both the linguistic features of academic oral language and social conventions that take place during these events (Ventola, Shalom \& Thompson, 2002). Academic conferences have been understudied (Robles Garrote, 2016), especially within the theoretical framework of Systemic Functional Linguistics (SFL). For this reason, researchers who investigate this topic have insufficient reference literature. This study contributes to the understanding of CPs by discussing theoretical and methodological aspects of the analysis of a corpus of 32 CPs given in Spanish in the United States to determine the generic structure or prototypical text structure within the SFL framework. The macrogenre and orbital structure constructs (Martin \& Rose, 2008) are proposed as ideal for the CPs' analysis. The analysis showed a particular generic structure for CPs, variation according to discipline and membership in the academic community. In this sense, this study helps to reduce the knowledge gap that exists about this topic and offers a methodological model to future SFL researchers.
\end{abstract}

Key Words: Systemic Functional Linguistics, generic structure, macro-genre, academic oral Spanish, Spanish in the USA.

\title{
INTRODUCCIÓN
}

Las conferencias académicas (CAs) comprenden congresos, jornadas, seminarios y simposios y, en el marco de estos, las ponencias, comunicaciones en un panel, plenarias, y mesas redondas, entre otros (Regueiro Rodríguez \& Sáez Rivera, 2013). Cada uno de estos contextos académicos imprime en los textos orales académicos que allí se producen una estructura y unas características léxico-gramaticales particulares. El modo presencial e interactivo de las CAs las diferencian de otros textos que se producen en el ámbito académico (Ventola et al., 2002; Hood \& Forey, 2005), puesto que la comunicación con la audiencia es inmediata (Viera, 2017, 2019). Asimismo, el texto de las CAs variará de acuerdo al contexto de situación y a la cultura específica que lo crea (Ventola et al., 2002), entendiéndose por cultura no solo el país o región sino también la comunidad profesional para quien la comunicación fue creada y que imponen convenciones estilísticas y estructurales a los textos académicos (Bolívar, 2004, 2005; Hyland, 2000; Suomela-Salmi \& Dervin, 2009; Orna-Montesinos, 2012). De igual manera, el tipo de interacción social entre los miembros de la comunidad académica se evidencia en el discurso académico (Bolívar, 2004; Swales, 2004; Rowley-Jolivet \& Carter-Thomas, 2005). El análisis de un texto de CA debe, por tanto, considerar el tipo de evento y la comunidad profesional que se quiere estudiar. A pesar de esto, las CAs se describen en manuales de consulta sin considerar la complejidad anteriormente señalada (Hyland \& Jiang, 2017).

Si bien las conferencias académicas (CAs) son una actividad de suma importancia profesional, se han investigado menos que los textos académicos escritos (Ventola, 2002; Robles Garrote, 2013, 2016; Hood, 2017). En nuestro conocimiento, son aún más escasos los estudios empíricos de CAs en español, y aún menos frecuentes con el marco teórico de la Lingüística Sistémico-Funcional (pero véase Hood \& Forey, 
2005). Esta carencia de bibliografía de referencia genera un desafío a los investigadores, especialmente en lo que concierne al español, ya que la mayoría de los estudios de CAs se centran en el idioma inglés. Por esto, los investigadores de CAs en español deben determinar cuestiones de índole teórico y metodológico sin tener estudios de referencia que los guíen. De esto se desprende la importancia de analizar corpus especializados que, mediante la comparación posterior de resultados, permitan encontrar patrones generales del discurso académico que se produce en las CAs en español.

El estudio que aquí se presenta, con el marco teórico de LSF, discute opciones teóricas y metodológicas pertinentes para el análisis de 32 comunicaciones, en español, en paneles realizados en los Estados Unidos por investigadores de Lingüística y Literatura. En particular presenta el análisis de potencial de estructura genérica (Martin, 2000/1997; Eggins \& Alcántara, 2002) de este corpus piloto. En este sentido, el análisis cuantitativo que aquí se presenta expande y profundiza información cualitativa originalmente presentada sobre este corpus en Viera (2017), haciéndose especial énfasis en la discusión de decisiones teóricas y metodológicas relacionadas con el uso del marco teórico de la Lingüística Sistémico-Funcional (LSF). Se intenta demostrar que la LSF ofrece herramientas teóricas y metodológicas que permiten el estudio de un texto tan variable a nivel de la estructura genérica como el de las CAs, que si bien planificados, deben ser actualizados en función de la audiencia, y son por tanto interactivos y dinámicos (Viera, 2017, 2019). Este marco teórico entiende los textos como estructuras socio-semióticas dinámicas, dependientes de la cultura que los crea, y que deben estudiarse en relación a un contexto sociolingüístico específico (Halliday \& Matthiessen, 2014; Martin, 2000/1997). Por ende, se busca aportar conocimientos tanto sobre los textos de las CAs como sobre la aplicabilidad de la LSF, y de determinados constructos teóricos, para el análisis discursivo de este tipo de texto oral de tanta importancia para la actividad académica. En particular, la determinación de la CA como un macro-género y la variación a nivel de estructura de acuerdo a la disciplina y membresía en la comunidad científica. El determinar esas posibles diferencias arroja información sobre la comunidad académica en cuestión: sus prioridades e intereses, y sus características idiosincráticas. En este sentido, los estudios de corpus piloto, como es el caso de este estudio, si bien arrojan información parcial, permiten la 'validación cuantitativa' que Moris y Navarro (2007) señalan como faltante en el campo de los estudios de estructura genérica ya que, de acuerdo a la teoría de género, si bien podríamos esperar que los textos de una CA no varíen demasiado en las diferentes comunidades académicas, es también esperable la variación y por ende, necesaria una descripción de los mismos para encontrar las similitudes o diferencias.

Este estudio ofrece una aproximación concreta a la manera en que la LSF puede usarse para entender los textos de las CAs como textos dinámicos. Este trabajo reviste 
importancia para quienes se interesan por aspectos del español académico oral, especialmente en lo que refiere a las CAs en los Estados Unidos, y a la idoneidad de la LSF como marco teórico que informe un análisis discursivo de género y registro. Las siguientes secciones detallan el marco teórico de este estudio, con especial énfasis en los constructos teóricos usados con el fin de describir el potencial de estructura genérico. La sección de resultados presenta los datos obtenidos del análisis de las ponencias de profesores y estudiantes. En principio, se presentan los resultados para todo el corpus con el fin de determinar una estructura común a ambas disciplinas y luego por separado para establecer diferencias.

\section{Marco teórico}

El lenguaje académico ha sido objeto de estudio de numerosas investigaciones que han concluido que el mismo se caracteriza por un tecnolecto propio de cada disciplina (tecnicidad) (Martin, 2001) y por el empleo de recursos gramaticales y discursivos específicos (Bolívar, 2004; Parodi, 2004; Hyland, 2009; Moyano, 2013, 2018) así como una forma de presentar la información en etapas secuenciales que es, en parte, gobernada por las convenciones estilísticas de cada disciplina.

La LSF presta especial atención al estudio de la lengua en contexto. En este marco teórico, el análisis de un texto implica analizar la interacción de tres variables que crean el 'registro': el 'campo', el 'tenor' y el 'modo' (Knapp \& Watkins, 2005; Halliday \& Matthiessen, 2014). Esto es, todo texto se crea atendiendo al 'campo' que es el tema o actividad central; al 'tenor' que expresa la relación entre los interlocutores, y al 'modo' que es el canal o forma en que se transmite o desarrolla ese texto. Estas variables registrales se instancian en el lenguaje, por tanto, el contexto de la situación comunicativa se instancia en el mismo. A su vez, las variables registrales se relacionan con las metafunciones ideacional, interpersonal y textual (Halliday \& Matthiessen, 2014) porque todos los usos que se hacen de la lengua redundan en tres: "una función de la expresión de la experiencia, una función de creación de relaciones interpersonales y una función de organización de la información" (Eggins \& Alcántara, 2002: 142). Sin embargo, existe solapamiento de las funciones del lenguaje. El registro del texto, entonces, se construye mediante una configuración determinada de elementos discursivos y lingüísticos.

El texto también instancia el potencial de significado del idioma para cumplir propósitos sociales. El concepto de 'género' que puede definirse como "un plano semiótico en el que se inserta el texto" (Matthiessen, Teruya \& Lam, 2010: 107, nuestra traducción) o "como configuraciones recurrentes de significados del registro, que representan o llevan a cabo las prácticas sociales de una cultura dada" (Moyano, 2018: 247) permite el estudio de dichos propósitos en la manera en que los textos se desarrollan o presentan en etapas. Los géneros se estructuran en fases o etapas que se orientan para "lograr el éxito de los objetivos sociales que lo caracterizan" (Moris \& 
Navarro, 2007: 9) y están supeditados a los acuerdos en los que los hablantes participan como integrantes de una misma comunidad (Eggins \& Alcántara, 2002; Moyano, 2013). Así, las diferentes comunidades profesionales organizarían la información que se presenta en las CAs de manera diferente porque la estructura de un texto constituye otra forma en que el contexto y la cultura se instancia en el mismo. Una discusión detallada de las diferentes aproximaciones al concepto de estructura genérica escapa al propósito de este estudio, pero véase Ciapuscio (2005); Moris y Navarro (2007); Moyano (2010, 2013, 2018).

El enfoque en la cultura que crea los textos y la determinación de la estructura de los tipos textuales ofrece ventajas al investigador que desea encontrar soluciones aplicables a contextos educativos. Una de estas soluciones es la identificación del potencial de la estructura genérica, lo que puede ser de utilidad para los novatos o aprendices en una comunidad científica o académica como guía para organizar sus textos (Martin \& Rose, 2007; Navarro \& Simões, 2019). La enseñanza de tipos textuales ajenos a las realidades cotidianas de los aprendices y la elaboración de propuestas pedagógicas eficaces que promuevan el desarrollo del lenguaje y la apropiación del discurso académico (Moyano, 2018) ha sido un gran aporte de la LSF. $\mathrm{El}$ análisis de la estructura genérica busca determinar las diferentes etapas constitutivas que deben estar presentes para que un texto se perciba como representante de un género dado, y establecer la secuencia de esas etapas (Taboada, 2004, 2011). Con respecto a las etapas funcionales que constituyen la estructura del género, existen elementos obligatorios, que definen el género discursivo, así como elementos opcionales, que pueden o no estar presentes. Rose (2007) utiliza de manera diferente los términos 'etapas' y 'fases' como manera de precisar la obligatoriedad e importancia de los elementos constitutivos de la estructura genérica. La subdivisión del texto en etapas funcionales nos permite la identificación de los micro-propósitos sociales y contribuye a la creación de significado mediante la estructuración lógica de la información, en este caso a nivel discursivo (Hood \& Forey, 2005). Sin embargo, esta concepción estática del género como una secuencia fija de etapas obligatorias no es compartida por todas las escuelas de la LSF, en particular, la escuela de Sídney que propone una concepción más fluida (Morris \& Navarro, 2007) que refleje la variación que imponen los contextos y las actualizaciones del texto en tiempo real.

\subsection{Macro-género y tipos de estructuras}

Sería posible afirmar que las comunicaciones orales en una CA tienen como fin primordial la divulgación de un trabajo académico y se las podría considerar un género puro. Sin embargo, este es un propósito organizador o macro-propósito. Existen otros propósitos sociales en las ponencias, tales como la construcción de una comunidad académica y discursiva y, el posicionamiento del orador en esa comunidad y con respecto al conocimiento generado en esa disciplina (Hyland, 2000, 2005). Por otro 
lado, Martin y Rose (2008) afirman que los géneros simples ocupan a lo máximo una página de extensión. Esto no quiere decir que la extensión sea el requisito obligatorio para clasificar un género, sino que las etapas necesarias para construir el conjunto de significados que representa un género se logran, por lo general, con pocas etapas. Ejemplos de géneros simples son la narración, el informe o los saludos, entre otros y para el discurso académico véase, entre otros, Moyano (2013) y Rose (2007). Todo texto más extenso implica recurrir a un mayor número de géneros simples que pasan a construir un macro-género, el cual es una estructura, dinámica y maleable (Martin, 1994; Martin \& Rose, 2008, Rose, 2014). Si se considera que la transcripción de una ponencia de 15 minutos resulta en unas 6 o 7 páginas de texto, la extensión misma nos da la pauta de que las ponencias se alejan de la extensión sugerida para un género puro (Martin \& Rose, 2008). Por extensión y nivel de complejidad en sus propósitos sociales, las comunicaciones orales en CAs pueden entenderse mejor como un macrogénero en el que los géneros simples se constituyen en las fases funcionales de esa estructura. Martin (1994) propone que en los macro-géneros existe un efecto de 'amplificación', efecto que compara con las relaciones lógicas que se establecen entre los complejos causales, por el cual un género es 'citado' por otro y es esta estructuración la que aporta la estabilidad, cohesión y coherencia estructural y de significado. Así, los diferentes géneros de un macro-género no hacen sino 'elaborar', 'extender' o 'destacar' el conjunto de significados creados por los otros géneros del sistema.

Por otra parte, Rose (2007) discute que es necesario considerar los modelos estructurales que mejor reflejen las diferencias entre los modos oral y escrito. Entre las representaciones posibles, la estructura orbital, definida como una estructura que se organiza alrededor de un núcleo de manera similar al sistema solar o atómico (Martin \& Rose, 2008) podría ser una mejor opción para describir la estructura de las ponencias. En este modelo existen núcleos que son elementos obligatorios de esa estructura mientras que los elementos satelitales son opcionales. Entendemos que esta representación visual puede arrojar una mejor interpretación de los resultados del análisis de estructura genérica de los textos de CAs en el cual el contexto situacional determina que exista un dinamismo diferente al del texto académico escrito que se caracteriza por ser planificado y sinóptico. Esta representación también permite plasmar que no todas las culturas o comunidades acuerdan una sucesión de fases idénticas ni tampoco coinciden en qué elementos constitutivos serán obligatorios u optativos (Martin, 2000/1997; Eggins \& Alcántara, 2002).

Por último, importa precisar el concepto de 'fase funcional' que Rose (2007: 4) define como "waves of information carrying pulses of field and tenor". Este autor diferencia las fases (phases) de las etapas (stages) y considera que las fases son elementos intermedios. De interés para este estudio es que tal distinción evidencia que la estructura genérica puede entenderse como un concepto más complejo que una mera concatenación de 
etapas esperables y reconocibles en una secuencia determinada. Las etapas que construyen el macro-género se constituirán por fases. Por lo discutido, el análisis de género en este estudio adopta el concepto de macro-género y de estructura genérica orbital. La siguiente sección detalla el procedimiento metodológico realizado.

\section{Marco metodológico}

\subsection{Corpus y participantes}

El corpus que se discute en este estudio es un corpus piloto de textos especializados, recopilados con el fin de informar estudios futuros, especialmente aquellos que combinen la LSF con las herramientas y tipo de análisis de la Lingüística de Corpus (McEnery \& Hardy, 2011). Los textos orales fueron creados en nueve conferencias distintas y por presentadores que trabajan en disciplinas diferentes, esto es, en Literatura y Lingüística. Los presentadores trabajan o estudian en programas universitarios de español en los Estados Unidos.

De igual manera, los participantes de este corpus reflejan diferentes grados de experiencia en la comunidad educativa. Esta decisión metodológica sobre la recolección de datos generó un corpus diverso, puesto que se buscaba encontrar los elementos comunes de las CAs en español en los Estados Unidos, y, por tanto, la variación de las fases de la estructura genérica era un resultado esperable. Sin embargo, generó inconvenientes a la hora de establecer la distribución obligatoria de la estructura, y, por ende, una fórmula única, lo que se subsanó al usar el constructo teórico del macro-género y la estructura orbital, decisión que se discute en los apartados siguientes.

Para la recolección de datos se siguieron los lineamientos exigidos por The Institutional Review Board (IRB) que es el organismo administrativo que rige las investigaciones que involucran seres humanos en los Estados Unidos. El corpus es el mismo que se utilizó en Viera (2017), pero ha sido etiquetado de nuevo, con aspectos diferentes de la anotación original que resultan de un proceso de validación más exhaustivo. El corpus exploratorio de este estudio comprende 32 comunicaciones orales en paneles en los Estados Unidos durante los años 2011-2012 en los espacios académicos que se detallan en la Tabla ${ }^{1}{ }^{1}$. 
Tabla 1. Participantes por evento.

\begin{tabular}{|c|c|}
\hline Número participantes & Evento \\
\hline 9 & UC Davis Colloquium (2011; 2012) \\
\hline 11 & Español en EE.UU. (2011; 2012) \\
\hline 7 & LASA (2012) \\
\hline 2 & Radical Politics (Berkeley) (2012) \\
\hline 1 & Other Iberias (2012) \\
\hline 1 & 33rd Cincinatti Conference (2012) \\
\hline 1 & NeMLA (2013) \\
\hline 32 & \\
\hline
\end{tabular}

Las presentaciones son en español y duran entre 13 y 20 minutos cada una, totalizando 9 horas y 33 minutos de grabación y 74.571 palabras. Solamente el texto oral (no el escrito o los medios audiovisuales) fue transcrito ortográficamente y anotado manualmente para el análisis. La siguiente Tabla 2 resume la información del corpus y de los participantes.

Tabla 2. Descripción general del corpus.

\begin{tabular}{|c|c|}
\hline Número de textos & $\mathbf{3 2}$ \\
\hline Número de palabras & 74,571 \\
\hline Horas & $9 \mathrm{~h} 33 \mathrm{~m}$ \\
\hline Modo y registro & Oral (exclusivamente) \\
& académico \\
& planificado \\
& monológico \\
\hline Disciplinas académicas & Literatura (18) \\
& Lingüística (14) \\
\hline Lengua materna o dominante & espanol (28) \\
& inglés (4) \\
\hline Rol universitario & Profesores 17: 10LIT; 7 LIN \\
& Estudiantes de postgrado \\
& 15: 8 LIT; LIN 7 \\
\hline
\end{tabular}

\subsection{Diseño de investigación y procedimiento de etiquetado}

Las presentaciones fueron video-grabadas por una de las autoras, transcriptas ortográficamente y manualmente etiquetadas para encontrar las diferentes macroetapas y fases de la estructura genérica. Estudios similares que analizan corpus a un nivel discursivo o pragmático han recalcado la dificultad en el proceso del etiquetado (Alsop \& Nesi, 2014; Alharbi \& Hain, 2016; Navarro \& Simões, 2019), especialmente en los casos en que no existen categorías o unidades ya definidas por estudios previos (Leech, McEnery \& Wynne, 1997). Uno de los aportes del estudio aquí presentado es el de contribuir en la definición de etapas y fases, pero, al carecer de estudios previos en este contexto que definan aspectos léxico-gramaticales de las mismas, las investigadoras desarrollaron su propio set de etiquetas. Para resolver el problema inherente de la ambigüedad en la etiquetación de unidades discursivas y pragmáticas, 
las investigadoras se basaron en su propia capacidad como miembros de la comunidad disciplinar de reconocer géneros y recursos retóricos propios de las ciencias y las humanidades. Un ejemplo de esto son las fases de saludo, agradecimiento, argumentación, narrativa, captatio benevolentiae, etc. Tal metodología es similar a la propuesta por anteriores investigaciones de comunicaciones orales profesionales, así como lo son las diferentes estrategias para delimitar fronteras (Rowley-Jolivet \& Carter-Thomas, 2005; Hood, 2017), como es el caso del discurso organizador o metadiscurso de los ponentes, esto es, cuando el ponente anuncia los segmentos de su presentación o su intención de comenzar a leer. Asimismo, marcadores del discurso que indican un cambio de etapa ('ahora procedemos a'... 'bueno', 'entonces') o cambios en el léxico, por ejemplo, mención de las palabras 'datos' o 'metodología' al pasar a la etapa de desarrollo, esto es una mayor tecnicidad comúnmente reservada a esta sección. De mayor importancia, en este caso, fueron los organizadores multimodales y visuales en las presentaciones PowerPoint, como lo son los títulos escritos, vídeos, imágenes y audios y cuyo uso ha sido reportado en investigaciones de las CAs como un elemento diferenciante de etapas (Rowley-Jolivet \& Carter-Thomas, 2005; Hood, 2017). Para reducir el error impuesto por la subjetividad en el etiquetado se implementó una revisión dual y discusión entre las investigadoras. Se mantuvieron los etiquetados en los que se llegaba al acuerdo. El proceso de etiquetado sigue a Eggins y Slade (1997) y Taboada (2004):

a) Delimitación de los propósitos del texto.

b) Delimitación de etapas señaladas en investigaciones previas: introducción, desarrollo, apertura, etc.

c) Reconocimiento de una unidad que cumpla un propósito social dentro de la etapa.

d) Identificación de ese propósito mediante etiquetado.

e) Identificación de las "fronteras" de etapas y fases.

f) Determinación de patrones recurrentes.

g) Especificación y cuantificación de etapas y fases obligatorias y opcionales.

h) Identificación de los géneros usados en estas etapas.

La etiquetación de etapas, fases y géneros fue informada por investigaciones previas de CAs y del discurso de la ciencia, especialmente en lo que hace a la denominación de las etapas y fases (Ventola, 2002; Carter-Thomas \& Rowley-Jolivet, 2003; Rowley-Jolivet \& Carter-Thomas, 2005; Maynard \& Leicher, 2007; Martin \& Rose, 2008; Nesi, Ahmad \& Ibrahim, 2009; Villar, 2011; Moyano, 2013; Robles Garrote, 2013; Alsop \& Nesi, 2014). En la denominación de 'etapas' y 'fases' como elementos diferentes se sigue a Rose (2007) y adaptamos esa nomenclatura para distinguir macro-etapas de etapas menos frecuentas (fases). 
Posterior al etiquetado, se usó herramientas de análisis de corpus (McEnery \& Hardy, 2011) para analizar la distribución de las fases en el corpus y de esta forma determinar aquellas que eran más frecuentes en todo el corpus y por disciplina. Como el propósito de este estudio es también la postulación del modelo orbital como idóneo para explicar los textos orales de las CAs, no se buscó establecer una fórmula estructural, sino los datos suficientes para determinar los núcleos obligatorios y opcionales. Para determinar la obligatoriedad de los elementos estructurales, consideramos los valores discutidos por Navarro y Simões (2019) y Navarro (2011), estableciendo los elementos con una recurrencia menor al $25 \%$ como no suficientemente estables. La frecuencia de distribución se clasificó de la siguiente manera: a) 25\%-45\%, ocasional; b) 46\%-70\%, frecuente; y c) $71 \%-100 \%$, obligatorio. Es de señalar que en principio se establecieron niveles más altos $(80 \%)$ para considerar los elementos como obligatorios, tal y como proponen Navarro y Simões (2019) en su análisis de un corpus de textos escritos. En nuestro corpus, por las características ya descritas anteriormente, un valor de $80 \%$ no permitía encontrar elementos obligatorios a nivel de las fases, aunque sí de las etapas. La siguiente tabla muestra el etiquetado de macro-etapas con su consiguiente descripción de propósito y función.

Tabla 3. Etapas funcionales usadas en el análisis de ponencias.

\begin{tabular}{|l|l|}
\hline \multicolumn{1}{|c|}{ Macro-etapa } & \multicolumn{1}{c|}{ Propósito y función } \\
\hline Apertura & $\begin{array}{l}\text { Captar la atención de la audiencia, posicionar el texto y al ponente en el } \\
\text { contexto académico. Puede realizarse por medio de saludo, agradecimiento, } \\
\text { exordio, pedido de benevolencia, justificación de la relevancia de la ponencia en } \\
\text { el contexto de la conferencia, humor, narrativas personales, etc. }\end{array}$ \\
\hline Introducción & $\begin{array}{l}\text { Orientar a la audiencia en lo que será tanto el tema de la ponencia, la } \\
\text { contextualización del mismo en el saber colectivo y explicitación del propósito } \\
\text { del trabajo en ese contexto. Se realiza por medio de anuncio de tema, estado de } \\
\text { la cuestión, contexto social, establecimiento de la relevancia de la investigación } \\
\text { realizada y planteamiento de tesis. }\end{array}$ \\
\hline Desarrollo & $\begin{array}{l}\text { Descripción de la metodología del análisis, discusión de resultados, análisis. } \\
\text { Obra como cierre a la argumentación presentada en defensa de la tesis } \\
\text { presentada o los resultados encontrados. Se conecta con el tema de la } \\
\text { presentación. }\end{array}$ \\
\hline Cierre & $\begin{array}{l}\text { Pone final al evento 'ponencia' y no es una conclusión lógica del tema tratado } \\
\text { ni se conecta con la tesis o pregunta de investigación del trabajo. Se realiza por } \\
\text { medio de saludos, agradecimiento, anuncio del fin de la ponencia o en la forma } \\
\text { de epílogo que destaca la importancia social del tema presentado y, en } \\
\text { ocasiones la inclusión de un pedido a la audiencia que se relaciona con el tema } \\
\text { presentado. }\end{array}$ \\
\hline
\end{tabular}

En el análisis de este corpus se usó AntConc 3.2.4 (Anthony, 2013). Este software posibilita encontrar las ocurrencias en contexto, cuantificar los casos y determinar los textos en donde se presentan los constitutivos de la estructura genérica. La búsqueda de tales elementos discursivos permite tanto encontrar rasgos comunes a todas las ponencias de este corpus como comparar patrones recurrentes. Las herramientas usadas fueron las siguientes: Concordance Tool, que encuentra los contextos en los que 
se dan los elementos etiquetados, y Concordance Plot, que encuentra los elementos etiquetados en el corpus y los cuantifica de acuerdo a los participantes. Siguen imágenes que ejemplifican la función de estas herramientas. Tras el etiquetado de etapas y fases en el corpus, el análisis con AntConc permitió establecer, mediante la cuantificación de la distribución de dichos elementos en el corpus, el patrón recurrente de elementos estables que conforman el texto de una CA, independientemente de la disciplina. Una vez determinada la estructura común, se analizó el cambio en la estructura en función de la disciplina y de la experiencia de los presentadores. Esto es, a los efectos de determinar la estructura obligatoria se analizó la estructura genérica construida por los miembros de la comunidad con mayor experiencia, puesto que son estos participantes quienes tienen el conocimiento de las prácticas discursivas propias de cada disciplina (Swales, 2004).

El fin de este trabajo es la determinación cuantitativa de la estructura genérica, y no así las características léxico-gramaticales de cada etapa que, por la complejidad del análisis cuantitativo de 32 ponencias, entendemos requeriría un estudio en sí mismo. Sin embargo, en la sección de resultados se resume de manera cualitativa los elementos en los que se encontró un patrón de distribución recurrente a simple vista. Como objetivo secundario, este estudio busca determinar la idoneidad del modelo de macro-género y de estructuras orbitales para la descripción de la ponencia en un panel de CAs.

\section{Resultados}

Como primer paso, interesaba encontrar los elementos estables recurrentes del macro-género. Esto es, una macro-estructura que refleje el potencial genérico de las CAs, independiente de la disciplina considerada. Para determinar qué macro-etapas se encuentran presentes en cada presentación, y cómo se diferencian entre ellas, se aplicó el método que se describe en la sección 3.2. de Metodología. En la Tabla 4 se presentan las etapas encontradas y su distribución porcentual en el corpus. En ella, 'N' equivale al número total de presentadores que estructuran el texto en esas etapas, y el 'porcentaje' se calcula en relación al total de 32 participantes.

Tabla 4. Etapas funcionales.

\begin{tabular}{|c|c|c|}
\hline Macro- etapa & Participantes $(\mathbf{N}=32)$ & Porcentaje \\
\hline Apertura & 28 & 87.5 \\
\hline Introducción & 32 & 100 \\
\hline Desarrollo & 32 & 100 \\
\hline Conclusión & 29 & 90.6 \\
\hline Cierre & 22 & 68.7 \\
\hline
\end{tabular}

Se puede ver que el 'cierre' no tiene, de acuerdo a los parámetros establecidos para esta investigación, un carácter obligatorio, aunque sí es muy frecuente. De igual 
manera, para las macro-etapas, la secuencia sí es estable y es la que se muestra en la Tabla 4. Interesa notar las etapas de 'apertura' y 'cierre' que no están presentes en los textos académicos escritos.

\subsection{Diferencias en la estructura genérica por disciplina}

A continuación, se presentan los datos del análisis de fases (en oposición a etapas) más frecuentes encontradas en el corpus analizado. La Tabla 5 que sigue recoge los datos obtenidos en el análisis del corpus de Literatura conformado por 18 ponentes. La columna de 'géneros' alude a los géneros cortos o simples que constituyen la fase. En la Tabla 5, 'N' equivale al número de presentadores y '\%” es el cálculo de la frecuencia de aparición de cada fase en relación al total de 18 ponentes de Literatura. Este cálculo ha sido redondeado para evitar decimales. Nótese que, por tratarse de fases, la secuencia en esta Tabla 5 no refleja lo encontrado en cada CA analizada. Esto es, la secuencia de las fases no es estable y puede variar con los presentadores, pero sí lo es su distribución en el corpus.

Tabla 5. Estructura genérica: Literatura.

\begin{tabular}{|l|l|l|l|l|}
\hline \multicolumn{1}{|c|}{$\begin{array}{c}\text { Macro- } \\
\text { etapa }\end{array}$} & \multicolumn{1}{|c|}{ Faces funcionales } & \multicolumn{1}{|c|}{ Géneros } & N=18 & \multicolumn{1}{c|}{$\%$} \\
\hline Apertura & Agradecimiento & Agradecimiento & 10 & 55 \\
\hline Apertura & Exordio & Exordio & 8 & 44 \\
\hline Introducción & Anuncio de tema & Anuncio & 12 & 66 \\
\hline Introducción & Contexto social e histórico & Narración; informe & 12 & 66 \\
\hline Introducción & Revisión bibliográfica & Descripción & 9 & 50 \\
\hline Introducción & Tesis & Argumentación & 15 & 83 \\
\hline Desarrollo & $\begin{array}{l}\text { Análisis / presentación de } \\
\text { evidencia }\end{array}$ & Informe; argumentación; cita & 13 & 72 \\
\hline Desarrollo & Revisión bibliográfica & $\begin{array}{l}\text { Argumentación; informe; análisis; } \\
\text { cita }\end{array}$ & 10 & 56 \\
\hline Desarrollo & $\begin{array}{l}\text { Presentación de evidencia (sin } \\
\text { citas) }\end{array}$ & Anécdota; narración & 8 & 44 \\
\hline Conclusión & Conclusión & Argumentación & 16 & 89 \\
\hline Cierre & Epílogo & Peroración; narración & 3 & 17 \\
\hline Cierre & Agradecimiento & Agradecimiento & 7 & 39 \\
\hline
\end{tabular}

De interés, las CAs de Literatura se caracterizan por un fuerte énfasis en la revisión bibliográfica que aparece tanto en la 'Introducción' como en el 'Desarrollo', aunque esta última se caracteriza por presentar investigaciones que corroboran lo hallado por el investigador en su propio análisis. La explicitación de la tesis también distingue estos textos.

La siguiente Tabla 6 recoge los resultados del análisis genérico de Lingüística. Presenta una estructura diferente a la de Literatura en lo que respecta a las fases, con la inclusión del informe de un proceso de investigación. Por motivos de espacio, la Tabla 6 muestra solo las fases funcionales frecuentes (estables) en este corpus; 'N' equivale al 
número de presentadores y '\%” es el cálculo de la frecuencia de aparición de cada fase en relación al total de 14 ponentes de Lingüística.

Tabla 6. Estructura genérica: Lingüística.

\begin{tabular}{|c|c|c|c|c|}
\hline Macro-etapa & $\begin{array}{c}\text { Fases } \\
\text { funcionales }\end{array}$ & Géneros & $\mathrm{N}=14$ & $\%$ \\
\hline Apertura & Saludo & Saludo & 5 & 36 \\
\hline Apertura & Exordio & Exordio & 5 & 35 \\
\hline Apertura & $\begin{array}{l}\text { La ponencia } \\
\text { como parte del } \\
\text { panel }\end{array}$ & Narración; argumento & 6 & 43 \\
\hline Introducción & $\begin{array}{l}\text { Anuncio de } \\
\text { tema }\end{array}$ & Anuncio & 12 & 86 \\
\hline Introducción & $\begin{array}{l}\text { Estado de la } \\
\text { cuestión }\end{array}$ & Descripción; informe & 10 & 71 \\
\hline Introducción & $\begin{array}{l}\text { Relevancia del } \\
\text { tema }\end{array}$ & Argumento & 8 & 57 \\
\hline Introducción & $\begin{array}{l}\text { Preguntas de } \\
\text { investigación }\end{array}$ & Preguntas & 9 & 64 \\
\hline Intro/Desarr. & Tesis explícita & Informe; argumento & 8 & 57 \\
\hline Desarrollo & Marco teórico & $\begin{array}{l}\text { Procedimiento; descripción; narración; } \\
\text { informe }\end{array}$ & 9 & 64 \\
\hline Desarrollo & $\begin{array}{l}\text { Metodología: } \\
\text { participantes }\end{array}$ & $\begin{array}{l}\text { Procedimiento; reporte; explicación; } \\
\text { narración }\end{array}$ & 9 & 64 \\
\hline Desarrollo & $\begin{array}{l}\text { Proceso de } \\
\text { investigación }\end{array}$ & $\begin{array}{l}\text { Descripción; análisis; reporte; explicación; } \\
\text { narración }\end{array}$ & 10 & 71 \\
\hline Desarrollo & $\begin{array}{l}\text { Análisis y } \\
\text { resultados }\end{array}$ & $\begin{array}{l}\text { Argumento; reporte; descripción; } \\
\text { explicación; narración }\end{array}$ & 14 & 100 \\
\hline Conclusión & Conclusión & Argumentación & 11 & 78 \\
\hline Cierre & Epílogo & Exhortación; argumentación & 10 & 71 \\
\hline Cierre & Agradecimiento & Agradecimiento & 8 & 57 \\
\hline
\end{tabular}

De interés, el 'Cierre' tiene una frecuencia de distribución mayor que en Literatura, pero no así la 'Apertura', y con excepción de las fases del desarrollo y conclusión, las fases constitutivas de las demás macro-etapas presentan mayor variación que en Literatura y pocas de ellas son muy frecuentes.

\subsection{Diferencias en la estructura genérica de acuerdo a la experiencia}

Por último, interesa destacar las etapas favorecidas por los oradores expertos puesto que esto muestra las etapas y fases favorecidas por quienes tienen mayor experiencia en las convenciones discursivas de sus disciplinas. Por esto, la Tabla 7 muestra los datos para los profesores de Literatura, esto es, 10 participantes, mientras que la Tabla 8 presenta los datos de los profesores de Lingüística. 'N' equivale al número de presentadores y '\%” es el cálculo de la frecuencia de cada fase en relación al total de ponentes que la usan. Se incluyen ejemplos. 
Tabla 7. Elementos estructurales: profesores de Literatura.

\begin{tabular}{|l|l|l|l|l|}
\hline \multicolumn{1}{|c|}{ Macro-etapa } & Fases funcionales & $\begin{array}{l}\text { Ponentes } \\
(\mathbf{N}=10)\end{array}$ & $\mathbf{\%}$ & \multicolumn{1}{|c|}{ Ejemplos } \\
\hline Apertura & $\begin{array}{l}\text { Agradecimiento; } \\
\text { humor, benevolencia }\end{array}$ & 9 & 90 & $\begin{array}{l}\text { Gracias, muchas gracias a todos } \\
\text { por estar aquí [P32] }\end{array}$ \\
\hline Introducción & Anuncio del tema & 9 & 90 & $\begin{array}{l}\text { Este trabajo es un primer } \\
\text { acercamiento a un pasaje de la } \\
\text { novela... [P9] }\end{array}$ \\
\hline Introducción & $\begin{array}{l}\text { Contexto social e } \\
\text { histórico del tema }\end{array}$ & 8 & 80 & $\begin{array}{l}\text { En 1927, México experimenta } \\
\text { los albores de... [P26] }\end{array}$ \\
\hline Introducción & Tesis explícita & 7 & 70 & $\begin{array}{l}\text { En esta comunicación es mi } \\
\text { intención señalar...[P20] }\end{array}$ \\
\hline Desarrollo & $\begin{array}{l}\text { Análisis de texto con } \\
\text { citas }\end{array}$ & 10 & 100 & $\begin{array}{l}\text {..que es lo que nos interesa ehh } \\
\text { y cito: [P19] }\end{array}$ \\
\hline Conclusión & $\begin{array}{l}\text { Conclusión del tema } \\
\text { Cierre }\end{array}$ & 9 & 90 & $\begin{array}{l}\text { En conclusión, veo la } \\
\text { importancia de este poema... } \\
\text { [P3] }\end{array}$ \\
\hline Agradecimiento & 7 & 70 & $\begin{array}{l}\text { Gracias [P32] } \\
\text { Y con eso... [P15] } \\
\text { O.K? [P2] }\end{array}$ \\
\hline
\end{tabular}

En la Tabla 7, nótese menor variabilidad de las fases que en las Tablas 5 y 6, y la importancia de la apertura. Asimismo, nótese que la 'Presentación de la evidencia' y conectar el texto de la ponencia con otros textos creados sobre el tema (Citas) tiene carácter obligatorio. Por último, la explicitación de la tesis es apenas obligatoria. El cierre, se vuelve más frecuente que en las Tablas 5 y 6 . Por ende, los profesores de Literatura, a diferencia de los estudiantes, valoran la inclusión de etapas y fases funcionales que realizan la función interpersonal del lenguaje. En otras palabras, el propósito social de la construcción directa e indirecta (revisión bibliográfica) parece ser un marcador de experiencia en el discurso oral académico.

Sigue la presentación de los datos correspondientes a la disciplina de Lingüística. El total de los ponentes de Lingüística que son profesores es de 7. En la Tabla 8 se presentan los elementos estructurales con una frecuencia alta en el corpus. ' $N$ ' equivale al número de presentadores y '\%” es el cálculo de la frecuencia de cada fase en relación al total de ponentes que la usan. Se incluyen ejemplos. 
Tabla 8. Elementos estructurales: profesores de Lingüística.

\begin{tabular}{|c|c|c|c|c|}
\hline Macro-etapa & Fases & $\begin{array}{l}\text { Ponentes } \\
(\mathrm{N}=7)\end{array}$ & $\%$ & Ejemplos \\
\hline Apertura & $\begin{array}{l}\text { Saludos; } \\
\text { agradecimiento; } \\
\text { Benevolencia; } \\
\text { Anuncio de tema }\end{array}$ & 5 & 71.4 & $\begin{array}{l}\text { entonces, hoy estaré hablando de que el } \\
\text { español académico... [P8] }\end{array}$ \\
\hline Introducción & $\begin{array}{l}\text { Revisión } \\
\text { bibliográfica }\end{array}$ & 7 & 100 & ... y Chomsky ha planteado que... [P27] \\
\hline Introducción & Relevancia del tema & 6 & 85.7 & $\begin{array}{l}\text { Pues hay que hacer investigación. De ahí } \\
\text { que, en este trabajo hay que seguir } \\
\text { investigando...[P27] }\end{array}$ \\
\hline Introducción & $\begin{array}{l}\text { Preguntas de } \\
\text { investigación }\end{array}$ & 5 & 71.4 & $\begin{array}{l}\text { ¿Cuál es la distribución de los tres } \\
\text { dialectos que afectan los factores } \\
\text { lingǘsticos? [P27] }\end{array}$ \\
\hline $\begin{array}{l}\text { Introducción/ } \\
\text { Desarrollo }\end{array}$ & Tesis explícita & 4 & 57 & $\begin{array}{l}\text { quiero ver si existen diferencias } \\
\text { lingüísticas sociales y la distribución y a } \\
\text { su vez proponer la propuesta de que... } \\
\text { [P27] }\end{array}$ \\
\hline Desarrollo & $\begin{array}{l}\text { Proceso de } \\
\text { investigación }\end{array}$ & 4 & 57 & $\begin{array}{l}\text { Ehh trabajamos con } 17 \ldots \text { escogimos, } 17 \\
\text { sujetos de... [P27] } \\
\text {...tuve que dejar mucha gente pero fui } \\
\text { capaz de recoger ahhm textos [P25] }\end{array}$ \\
\hline Desarrollo & Resultados & 7 & 100 & Y veamos los resultados. [P27] \\
\hline Conclusión & Conclusión del tema & 5 & 71.4 & $\begin{array}{l}\text { Ha llegado la hora de presentar unas } \\
\text { breves conclusiones [P13] }\end{array}$ \\
\hline Cierre & $\begin{array}{l}\text { Epílogo; } \\
\text { Agradecimiento } \\
\text { Anuncio del fin }\end{array}$ & 6 & 85.7 & $\begin{array}{l}\text {...esto sale de lo que estaba presentando, } \\
\text { solamente que me moría por compartirlo } \\
\text { con ustedes [P14] } \\
\text { Y, es todo. [P29] }\end{array}$ \\
\hline
\end{tabular}

Por último, al igual que lo observado en las ponencias de Literatura, los profesores de Lingüística dan mayor prominencia a las etapas de 'Apertura' y 'Cierre'. En cambio, las fases de 'Informe del proceso', 'Tesis' y la 'Revisión bibliográfica' son menos frecuente que lo encontrado en las Tablas 5 y 6 , que incluyen a estudiantes. Nótese que la fase 'Preguntas de investigación' puede permitir la inferencia de la tesis y esta es frecuente entre los profesores. También es obligatoria la fase de 'Resultados'. Esto parece indicar un mayor énfasis en el producto final de la investigación. Lo anterior demuestra que los profesores de este corpus manejan la diferencia entre el proceso de estructuración del documento escrito de la investigación, en donde las fases iniciales son aquellas en donde se establece la credibilidad mediante la presentación del contexto y metodología detallada del estudio, y la comunicación oral de la misma en la cual, por las exigencias del procesamiento de la información, se requiere un enfoque en los resultados y menor énfasis en las etapas de establecimiento de la credibilidad. Al menos, esto es lo sugerido para el contexto de los Estados Unidos en lo que hace a la divulgación científica (Baron, 2010; Compass Science Communication, 2017). Al enfocarse en los resultados de la investigación y darle preponderancia a las fases que 
tienen un propósito social interpersonal, el investigador se posiciona como un experto que puede prescindir de establecer su credibilidad y que apuesta al diálogo entre pares.

\subsection{Patrones léxico-gramaticales y discursivos}

La siguiente es una descripción cualitativa de los patrones de elementos lingüísticos encontrados en los niveles discursivo y léxico-gramatical.

Tabla 9. Patrones discursivos y léxico-gramaticales.

\begin{tabular}{|c|c|}
\hline Macro-etapa & Patrones \\
\hline Apertura & $\begin{array}{l}\text { Errores, falsos arranques. } \\
\text { Léxico coloquial o no-técnico. } \\
\text { Elementos coloquiales: uso de diminutivos y marcadores } \\
\text { discursivos conversacionales (entonces; también; bueno; es decir; } \\
\text { pues); continuativos. } \\
\text { Marcadores de la comprensión y consenso: ¿no?; ¿ ¿í?; ¿ ¿verdad? } \\
\text { Uso de vocativos (nombres de colegas presentes). } \\
\text { Texto interactivo: uso de pronombres de primera y segunda } \\
\text { persona; preguntas retóricas; preguntas directas; textos } \\
\text { congruentes. } \\
\text { Modalización. } \\
\text { Cláusulas con agentes activos unidas en relaciones paratácticas. } \\
\text { Estrategias de repetición y aposición de información mediante } \\
\text { relaciones de proyección. } \\
\text { Narrativas personales. } \\
\text { Diálogo directo con la audiencia. }\end{array}$ \\
\hline Introducción & $\begin{array}{l}\text { Modo declarativo. } \\
\text { Nominalización. } \\
\text { Estrategias de despersonalización: ‘se' impersonal y pasivas reflejas; } \\
\text { pasivas; pronombres impersonales; plural de modestia o } \\
\text { mayestático; metonimia; otras expresiones impersonales. } \\
\text { Incongruencia gramatical: metáforas gramaticales. } \\
\text { Procesos verbales (habla, dice), mentales (piensa, considera, se } \\
\text { centra) y existenciales (hay). Verbos materiales conjugados en } \\
\text { pasado. } \\
\text { Heteroglosia: citas directas. }\end{array}$ \\
\hline Desarrollo & $\begin{array}{l}\text { Modo declarativo. } \\
\text { Nominalización. } \\
\text { Despersonalización. } \\
\text { Lenguaje técnico. } \\
\text { Lenguaje descriptivo. } \\
\text { Procesos relaciones, materiales y mentales (en ese orden). } \\
\text { Incongruencia gramatical: uso de metáforas gramaticales. } \\
\text { Procesos verbales (habla, dice), mentales (piensa, considera, se } \\
\text { centra) y existenciales (hay). Verbos materiales conjugados en } \\
\text { pasado. } \\
\text { Heteroglosia: citas directas. } \\
\text { Aumento de relaciones lógico-semánticas de hipotaxis. } \\
\text { Expansión de la frase nominal. }\end{array}$ \\
\hline Conclusión & Se repiten los patrones encontrados en la introducción. \\
\hline Cierre & Se repiten los patrones encontrados en la introducción. \\
\hline
\end{tabular}

Como se puede observar en la Tabla 9, la macro-etapa de Apertura incorpora elementos más personales e interactivos, respondiendo a una necesidad de establecer 
la relación entre el/la ponente y la audiencia. Vemos elementos léxico-gramaticales que corresponden a la función interpersonal de la lengua, como son los vocativos, la modalización y el uso de elementos continuativos. En la etapa de la introducción comienza el uso del lenguaje académico, con la presencia de nominalizaciones y estrategias que reorientan el lenguaje hacia un modo más impersonal, con énfasis en elementos ideacionales. Esta tendencia se profundiza en la etapa de desarrollo, que además incorpora estrategias de hipotaxis en la estructura de las oraciones y un mayor uso del lenguaje técnico. Estas relaciones se utilizan para enlazar ideas complejas, propias del desarrollo de las ideas. Finalmente, la 'Conclusión' y el 'Cierre' progresivamente vuelven a un estilo más coloquial, de reconexión con la audiencia. La descripción de estas etapas en lo que hace a los elementos léxico-gramaticales que las crean excede el propósito de este trabajo que se enfoca en la identificación y la cuantificación del potencial de estructura genérica de CAs pero entendemos que lo hallado puede informar futuros estudios cuantitativos con ese fin.

La siguiente Figura 1 representa la cohesión estructural encontrada en las CAs de este corpus, así como las fases más frecuentes de la estructura orbital. En lo que respecta a la estructura orbital, las macro-etapas funcionan como los núcleos a los que se asocian las fases funcionales, pero el orden en el que se presentan en la Figura 1 no representa la secuencia obligatoria de las mismas. Esto es, el análisis ha dejado claro que, en lo que compete a las fases funcionales de las CAs de este corpus, existe gran variación en lo que refiere a la secuencia de las fases. Por tanto, este análisis ha dejado claro que el potencial genérico varía de acuerdo a la disciplina y, en ocasiones la experiencia y, por tanto, que tiene una estructura más flexible y maleable que el texto escrito, que puede ser captada en el modelo orbital de macro-género. 


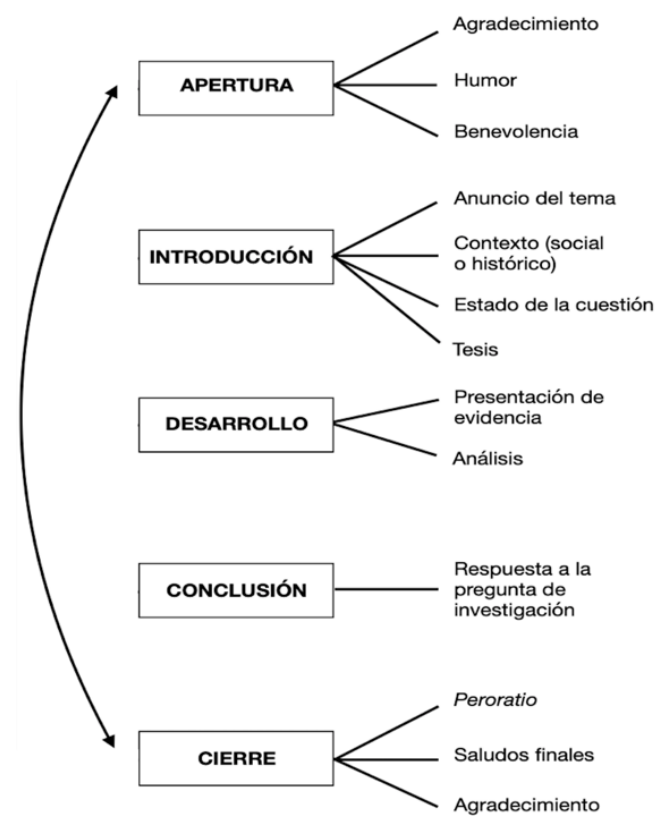

Figura 1. Estructura genérica.

\section{Discusión}

El análisis de género permitió corroborar lo encontrado en Viera (2017) de que las ponencias de este corpus son un macro-género que se estructura en macro-etapas estables: a) apertura, b) introducción, c) desarrollo, d) conclusión y, e) cierre. Estas etapas se organizan secuencialmente en el orden presentado y son de carácter obligatorio para ambas disciplinas, con excepción del 'cierre' que, probablemente por motivos de tiempo no siempre está presente. Esta macro-estructura se traduce en la siguiente fórmula estructural: \{Apertura ${ }^{\wedge}$ Introducción ${ }^{\wedge}$ Desarrollo ${ }^{\wedge}$ Conclusión ${ }^{\wedge}$ (Cierre) $\}$. En esta fórmula, el símbolo “^” representa el orden secuencial favorecido y las llaves "\{ "de comienzo y cierre manifiestan los elementos que forman parte de la estructura general del género mientras que los paréntesis marcan elementos opcionales (Eggins \& Alcántara, 2002). En lo que concierne a las fases que integran cada macroetapa, la siguiente es una fórmula que se desprende del análisis de este corpus de profesores y estudiantes de posgrado (pero recuérdese que la secuencia no es lineal): $\{\text { (Agradecimiento })^{\wedge}$ Anuncio y presentación del tema ${ }^{\wedge}$ Contexto social/histórico ${ }^{\wedge}$ $($ Revisión bibliográfica $) \wedge($ Tesis $) \wedge$ Análisis y argumentación ^ (Relevancia del tema $) \wedge$ Conclusión $\wedge$ Cierre $\}$. Dichas fases se construyen con géneros simples, como el saludo, agradecimiento, el informe y la narrativa personal, entre otros. Esto corrobora que las CAs son un macro-género en el cual las etapas que cumplen un propósito social interpersonal son favorecidas, así como las fases y géneros simples que amplifican y extienden esa función. Esto concuerda con lo encontrado por Thompson (2002: 148), quien afirma que los ponentes recurren a géneros narrativos para contar el proceso de investigación y estructuran su texto de manera tal que logren la atención 
del público presente usando: "techniques such as storytelling, metaphor and constructed dialogue". Entender las CAs como un macro-género es vital para un estudio cabal de las mismas.

Los elementos léxico-gramaticales encontrados reflejan los procesos de la estructura misma, esto es, son configuraciones recurrentes de significados que llevan a cabo los propósitos sociales de cada macro-etapa. De interés, como elemento diferenciante del texto escrito, este análisis evidencia una estructuración de macroetapas iniciales y finales con un propósito social interpersonal y en las cuales el lenguaje académico se aproxima al lenguaje coloquial. Esta aproximación no es un abandono del lenguaje académico, sino una adecuación a las exigencias del contexto y a los propósitos sociales de la negociación de significados con la comunidad académica allí presente. Existe, entonces, a nivel estructural, un efecto de amplificación en el cual la etapa inicial de apertura es 'destacada', en términos de Martin (1994), por la de cierre, lográndose así la cohesión del macro-género. De igual manera, en el análisis presentado anteriormente, es posible ver la manera en que las fases funcionales de esta estructura funcional, tal y como Rose (2007) las describe, aportan flujos de información y significado que construyen el campo, el tenor y el modo en cada etapa. Así, como apunta Hood (2017), la estructura genérica identificada en este estudio constituye un patrón del registro académico.

Por último, el análisis permitió identificar diferencias a nivel de las fases en relación con la experiencia en la comunidad académica y en la disciplina considerada. Las fases funcionales en el corpus de profesores de Lingüística responden al propósito social de informar sobre los resultados de una investigación y, por tanto, no sorprende que sea en el desarrollo donde se distingue de las ponencias de Lingüística.

\section{CONCLUSIÓN}

Por lo discutido en las secciones anteriores, el marco de la LSF posibilita analizar el potencial de estructura genérica de las comunicaciones orales de las CAs. Corpus especializados como el de este estudio permiten establecer las diferencias que los contextos de las diferentes disciplinas imparten al texto, así como identificar la manera en que las comunidades académicas crean textos que les permiten informar, relacionarse y construir la comunidad discursiva. Los propósitos vinculares son más relevantes en las CAs y se instancia en las etapas de 'Apertura' y 'Cierre' obligatorias o muy frecuentes en este corpus, especialmente en profesores. La capacidad de instanciar esta interacción en la organización del discurso, sin por ello abandonar el registro académico, distingue estos textos orales de otros textos. En concordancia con estudios anteriores de CAs (Hood \& Forey, 2005), este trabajo destaca la importancia de los elementos del lenguaje que manifiestan el propósito social de la vinculación con la audiencia y el posicionamiento del ponente ante el conocimiento colectivo de su 
comunidad académica. Las etapas de 'Apertura' y 'Cierre' pueden ofrecer oportunidades de análisis con ese fin, pero nótese que este tipo de significado interpersonal se construye a lo largo de toda la presentación y puede ser encontrado en las fases y los géneros simples que constituyen la macro-estructura.

En lo que respecta a aspectos metodológicos, este trabajo ofrece pautas para obtener un modelo de estructura genérica aplicable a diferentes contextos de situación. La variabilidad de los textos del corpus puede resolverse al considerar las CAs como un macro-género y usarse un modelo de estructura orbital. En esta estructura, las macro-etapas funcionales son estables y ofrecen la estructura requerida para que los textos puedan reconocerse como comunicaciones orales académicas en una comunidad dada, y obran como los núcleos de la estructura orbital. Por tanto, el modelo orbital y el concepto de macro-género resuelven dificultades del análisis, como el hecho de que el orden de aparición de las fases que conforman las etapas funcionales varíe en mayor medida que para los textos escritos. Teniendo esto en cuenta, es posible llegar a fórmulas estructurales a nivel de las macro-etapas. La diferencia clave con otros modelos radica en que al analizar una CA no se busque encontrar una secuencia obligatoria de fases funcionales. En la medida en que el ponente estructure su presentación en función de los núcleos constituidos por las macro-etapas estables, la comunidad profesional reconocerá al macro-género como correctamente construido para cumplir los propósitos específicos de la disciplina. Asimismo, las macro-etapas se relacionan con los propósitos sociales de las CAs que son: a) el establecimiento de relaciones interpersonales con la comunidad académica y b) el informe de una investigación académica.

Este estudio ha podido determinar que las ponencias son textos más dependientes del contexto que los textos académicos escritos. En este sentido, sería de interés establecer la variación de la estructura genérica en diferentes comunidades académicas de habla hispana mediante estudios similares.

\section{REFERENCIAS BIBLIOGRÁFICAS}

Alsop, S. \& Nesi, H. (2014). The pragmatic annotation of a corpus of academic lectures. En Calzolari, N. K. Choukri, T. Declerck, H. Loftsson, B. Maegaard, J. Mariani, A. Moreno, J. Odijk \& S. Piperidis (Eds.), Proceedings of LREC 2014, Ninth International Conference on Language Resources and Evaluation (pp. 15601563). Reykjavik, Iceland.

Alharbi, G. \& Hain, T. (2016). The OpenCourseW are Metadiscourse (OCWMD) Corpus. LREC [en línea]. Disponible en: http://www.lrecconf.org/proceedings/lrec2016/pdf/1085_Paper.pdf 
Anthony, L. (2013). AntConc (Version 3.2.4) [Computer Software]. Tokyo, Japan: Waseda University [en línea]. Disponible en: http://www.antlab.sci.waseda.ac.jp/

Baron, N. (2010). Escape from the Ivory Tower: A guide to making your science matter. Island Press: Washington, USA.

Bolívar, A. (2004). Análisis crítico del discurso de los académicos. Revista Signos. Estudios de Lingüística, 37(55), 7-18 [en línea]. Disponible en: https://dx.doi.org/10.4067/S0718-09342004005500001

Bolívar, A. (2005). Tradiciones discursivas y construcción del conocimiento en las humanidades. Signo y Seña, 14, 67-91.

Carter-Thomas, S. \& Rowley-Jolivet, E. (2003). Analysing the scientific conference presentation (CP): A methodological overview of a multimodal genre. ASp, La Revue du GERAS, 39(40), 59-72.

Ciapuscio, G. (2005). La noción de género en la Lingüística Sistémico Funcional y en la Lingüística Textual. Revista Signos. Estudios de Lingü̈stica, 38(57), 31-48.

COMPASS Science Communication, Inc. (2017). The Message Box Workbook [en línea]. Disponible en: https://www.compassscicomm.org/

Eggins, S. \& Alcántara, F. (2002). Introducción a la Lingüistica Sistémica. Logroño: Universidad de La Rioja.

Eggins, S. \& Slade, D. (1997). Analysing casual conversation. Londres: Cassell.

Halliday, M. A. K. \& Matthiessen, C. M. I. M. (2014). Halliday's Introduction to Functional Grammar (4ta. edición). Abingdon: Routledge.

Hyland, K. (2000). Disciplinary discourses: Social interactions in academic writing. Harlow, Ingalterra, Nueva York: Longman.

Hyland, K. (2005). Stance and engagement: A model of interaction in academic discourse. Discourse Studies, 7(2), 173-192.

Hyland, K. (2009). Academic discourse: English in a global context. Londres: Continuum.

Hyland, K. \& Jiang, F. (2017). Is academic writing becoming more informal? English for Specific Purposes, 45, 40-51.

Hood, S. (2017). Live lectures: The significance of presence in building disciplinary knowledge. Onomázein, (Número especial), 179-208. fecha de Consulta 26 de Septiembre de 2020]. ISSN: 0717-1285 [en línea]. Disponible en: https:/ / www.redalyc.org/articulo.oa?id=1345/134550067004 
Hood, S. \& Forey, G. (2005). Introducing a conference paper: Getting interpersonal with your audience. JE AP Journal of English for Academic Purposes, 4(4), 291-306.

Knapp, P. \& Watkins, M. (2005). Genre, text, grammar: Technologies for teaching and assessing writing. Sydney: UNSW Press.

Leech, G., McEnery, A. M. \& Wynne, M. (1997). Further levels of annotation. En R. Garside, G. Leech \& A. McEnery (Eds.), Corpus Annotation: Linguistic Information from Computer Text Corpora (pp. 85-101). Londres: Longman.

Matthiessen, C. M. I. M., Teruya, K. \& Lam, M. (2010). Key terms in Systemic Functional Linguistics. Nueva York/Londres: Continuum.

Martin, J. R. (1994). Macro-genres: The ecology of the page. Network, 21, 29-52.

Martin, J. R. (2000/1997). Analysing genre: Functional perspectives. En F. Christie \& J. R. Martin (Eds.), Genre and Institutions: Social processes in the workplace and school (pp. 3-39). Londres: Continuum.

Martin, J. R. (2001). Technicality and abstraction: Language for the creation of specialized texts. En A. Burns \& C. Coffin (Eds.), Analysing English in a global context: $A$ reader (pp. 211-228). Londres, Nueva York: Routledge in association with Macquarie University and The Open University.

Martin, J. R. \& Rose, D. (2007). Working with discourse: Meaning beyond the clause. Londres: Continuum.

Martin, J. R. \& Rose, D. (2008). Genre relations: Mapping culture. Londres, Oakville, CT: Equinox.

Maynard, C. \& Leicher, S. (2007). Pragmatic annotation of an academic spoken corpus for pedagogical purposes. En E. Fitzpatrick (Ed.), Corpus Linguistics beyond the word: Corpus research from phrase to discourse. Ámsterdam: Rodopi.

McEnery, T. \& Hardie, A. (2011). Corpus linguistics: Method, theory and practice (Cambridge textbooks in linguistics). Cambridge: Cambridge University Press.

Moris, J. P. \& Navarro, F. (2007). Género y registro en la Lingüística Sistémico Funcional. Un relevo crítico. En Actas del I Coloquio Argentino del Grupo ECLAR Texto y Género, Universidad Nacional de la Plata, Argentina.

Moyano, E. I. (2010). Aportes del análisis de género y discurso a los procesos de enseñanza y aprendizaje escolares: Las ciencias biológicas y la historia. Discurso \& Sociedad, 4(2), 294-331. 
Moyano, E. I. (2013). Una didáctica de las ciencias basada en los géneros textuales: Acceso a las disciplinas a través de la apropiación de su discurso. En E. Moyano (Ed.), Aprender ciencias y bumanidades: Una cuestión de lectura y escritura. Aportes para la construcción de un programa de inclusión social a través de la educación lingüistica (pp. 109-155). Los Polvorines: Uiversidad Nacional de General Sarmiento.

Moyano, E. I. (2018). La enseñanza de la lectura y la escritura académicas mediante programas a lo largo del curriculum universitario: Opción teórica, didáctica y de gestión. DELTA: Documentação de Estudos em Lingüistica Teórica e Aplicada, 34(1), 235-267 [en línea]. Disponible en: https://dx.doi.org/10.1590/0102445074896274115057

Navarro, F. (2011). Análisis histórico del discurso. La evaluación en las reseñas del Instituto de Filología de Buenos Aires (1939-1989). Tesis doctoral, Universidad de Valladolid. Facultad de Filosofía y Letras, Valladolid, España.

Navarro, F. \& Simões, A. C. (2019). Potencial de Estructura Genérica en tesis de ingeniería eléctrica: Contrastes entre lenguas y niveles educativos. Revista Signos. Estudios de Lingüística, 52(100), 306-329.

Nesi, H., Ahmad, U. \& Ibrahim N. M. (2009). Pragmatic annotations in spoken international corpus. Ponencia presentada en American Association of Corpus Linguistics Annual Meeting (AACL 2009). 11-13 October 2009. University of Alberta, Edmonton, Canadá [en línea]. Disponible en: https:/ / sites.ualberta.ca/ aacl2009/PDFs/NesiAhmadIbrahim2009AACL.p df

Orna-Montesinos, C. (2012). Constructing professional discourse: A multiperspective approach to domain-specific discourses. Newcastle upon Tyne: Cambridge Scholars.

Parodi, G. (2004). Textos de especialidad y comunidades discursivas técnicoprofesionales: una aproximación basada en corpus computarizado. Estudios filológicos, 39, 7-36 [en línea]. Disponible en: https://dx.doi.org/10.4067/S0071-17132004003900001

Regueiro Rodríguez, M. L \& Sáez Rivera, D. (2013). El español académico: Guía práctica para la elaboración de textos académicos. Madrid: Arco.

Robles Garrote, P (2016). Aportaciones de la Lingüística de Corpus al estudio de la conferencia como género académico de divulgación científica. CHIMERA: Romance Corpora and Linguistic Studies, 3, 1-21.

Robles Garrote, P. (2013). La conferencia como género monológico: Análisis macroestructural en español e Italiano. Boletín de Filología, 48, 127-146. 
Rose, D. (2007). Reading genre: A new wave of analysis. Linguistics and the Human Sciences, $2,1$.

Rose, D. (2014). Analysing pedagogic discourse: An approach from genre and register. Functional Linguistics 1(11), 1-13 [en línea]. Disponible en: https:/ / doi.org/10.1186/s40554-014-0011-4

Rowley-Jolivet, E. \& Carter-Thomas, S. (2005). The rhetoric of conference presentation introductions: context, argument and interaction. International Journal of Applied Linguistics, 15(1), 45-70.

Suomela-Salmi, E. \& Dervin, F. (Eds.) (2009). Cross-linguistic and Cross-cultural perspectives on academic discourse. Ámsterdam: John Benjamins.

Swales, J. M. (2004). Research genres: Explorations and applications. Cambridge, UK: Cambridge University Press.

Taboada, M. (2011). Stages in an online review genre. Text and Talk, 31(2), 247-269.

Taboada, M. (2004). The genre structure of bulletin board messages. Text Technology, $13(2), 55-82$.

Thompson, S. (2002). As the story unfolds: The use of narrative in research presentations. En E. Ventola, C. Shalom \& S. Thompson (Eds.), The language of conferencing (pp. 147-167). Frankfurt am Main; Nueva York: Peter Lang.

Ventola, E. (2002). Why and what kind of focus on conference presentations? En E. Ventola, C. Shalom \& S. Thompson (Eds.), The Language of Conferencing (pp. 15-50). Frankfurt am Main; Nueva York: Peter Lang.

Ventola, E., Shalom, C. \& Thompson, S. (2002). The Language of Conferencing. Frankfurt am Main; Nueva York: Peter Lang.

Viera, C. (2017). Genre and register variation: Academic conference presentations in Spanish in the United States. En J. Colomina-Alminana (Ed.), Contemporary Advances in Theoretical and Applied Spanish Linguist Variation (pp. 148-161). Athens, Ohio: Ohio State University Press.

Viera, C. (2019). La informalidad como recurso en el español académico de los Estados Unidos. En G. L. Thompson \& S. Alvord, (Eds.), Contact, Community, and Connections: Current Approaches to Spanish in Multilingual Populations (pp. 5982). Wilmington, DE: Vernon Press.

Villar, C.M. (2011). Las presentaciones académicas orales de los estudiantes alemanes de E/LE. Del discurso monológico al dialógico. Revista Nebrija de Lingüística Aplicada, 10(5), 130-172. 


\section{* AGRADECIMIENTOS}

Las autoras agradecen a los participantes de este corpus, así como a los revisores anónimos cuyos comentarios y sugerencias fueron fundamentales en el proceso de creación de este artículo.

\section{NOTA}

${ }^{1}$ Esta Tabla fue originalmente publicada en Viera (2017: 66) y se reproduce con permiso de la editorial. 